\title{
Optimized $\delta$ expansion for the Walecka model
}

\author{
G. Krein ${ }^{a}$, D.P. Menezes ${ }^{b}$, and M.B. Pinto ${ }^{c}$ \\ ${ }^{a}$ Instituto de Física Teórica, Universidade Estadual Paulista, \\ Rua Pamplona 145, 01405-900 São Paulo - S.P., Brazil \\ ${ }^{b}$ Departamento de Física, Universidade Federal de Santa Catarina, \\ 88.040-900 Florianópolis - S.C., Brazil \\ ${ }^{c}$ Laboratoire de Physique Mathématique - Université de Montpellier II \\ CNRS-URA 768 - 34095 Montpellier Cedex 05, France
}

\begin{abstract}
The optimized $\delta$-expansion is used to study vacuum polarization effects in the Walecka model. The optimized $\delta$-expansion is a nonperturbative approach for field theoretic models which combines the techniques of perturbation theory and the variational principle. Vacuum effects on self-energies and the energy density of nuclear matter are studied up to $\mathcal{O}\left(\delta^{2}\right)$. When exchange diagrams are neglected, the traditional Relativistic Hartree Approximation (RHA) results are exactly reproduced and, using the same set of parameters that saturate nuclear matter in the RHA, a new stable, tightly bound state at high density is found.
\end{abstract}


Although hadrons are not elementary particles, the use of renormalizable relativistic quantum field models employing pointlike hadrons, such as the Walecka model [1], for studying the properties of hadronic matter at high density and/or temperature is important in several aspects. Perhaps one of the most important aspects is the necessity of understanding the vacuum of such models, since one expects that the description of highly excited matter in terms of such models must breakdown at some scale and then quark-gluon degrees of freedom must be invoked for a proper treatment of the system. Quantum fluctuations in the Walecka model have been studied at the Hartree level [2], but severe difficulties arise when nonperturbative exchange diagrams (Fock diagrams) are considered. Although Bielajew and Serot [3] have set up an appropriate framework for the renormalization of the Hartree-Fock (HF) equations, no explicit calculation employing this has been performed so far. Bearing in mind the difficulties associated with the renormalization within the HF approximation we give in this Letter the first step towards the implementation of a different nonperturbative approach for studying vacuum fluctuations in hadronic models. Namely, we use the $\delta$ expansion [四]; more specifically we use the optimized linear $\delta$-expansion $[5]$.

The standard application of the linear $\delta$-expansion [5, 6, 7] to a theory with action $S$ starts with an interpolation defined by

$$
S(\delta)=(1-\delta) S_{0}(\mu)+\delta S=S_{0}(\mu)+\delta\left[S-S_{0}(\mu)\right]
$$

where $S_{0}(\mu)$ is the action of a solvable theory. The action $S(\delta)$ interpolates between the solvable $S_{0}(\mu)$ (when $\delta=0$ ) and the original $S$ (when $\delta=1$ ). Since $S_{0}$ is quadratic in the fields, arbitrary parameters $(\mu)$ with mass dimensions are required for dimensional balance. The evaluation of a physical quantity $P$ is performed by considering the term $\delta\left[S-S_{0}(\mu)\right]$ as a perturbation whose order is labeled by $\delta$ which is set to unity at the end. In practice, the perturbative expansion in powers of $\delta$ will be truncated at a given order implying that the quantity $P$ will have a residual dependence on the unknown parameters. Since $\mu$ does not belong to the original theory it will have to be fixed according to some criterion and different methods have been proposed. Among them the Principle of Minimal Sensitivity (PMS) [8] offers a particularly attractive way of optimizing the theory by requiring $P(\mu)$ to be evaluated at the point where it is less 
sensitive to small variations of $\mu: \partial P(\mu) / \partial \mu=0$. With this variational procedure $\mu$ becomes a function of the original parameters of the theory yielding non-perturbative results.

The different forms of the $\delta$ expansion have been successfully applied to many different problems in quantum mechanics, particle theory, statistical physics and lattice field theory, and its convergence has been recently proved for quantum mechanical problems [9]. More recently, the optimized linear $\delta$-expansion was used for applications in $\Phi_{4}^{4}$, QED and Yang-Mills theories [10]. In a recent paper [7] it was demonstrated that in truncating the $\delta$ expansion at $\mathcal{O}\left(\delta^{2}\right)$, and neglecting vacuum effects, one can readily reproduce the results of the standard self-consistent Dirac-Hartree-Fock approximation [2] (in this approximation vacuum effects are also neglected) for the equation of state of nuclear matter. In this Letter we go one step further by including vacuum effects to $\mathcal{O}\left(\delta^{2}\right)$ and demonstrate that one can reproduce the standard relativistic Hartree results (RHA). In addition, from the PMS applied to the renormalized energy density of nuclear matter, a new stable state is found at high density. One of the stengths of the $\delta$-expansion is that one can proceed to beyond leading order with considerably less computational effort than in the traditional HF approximation since in one deals in this approach with a finite number of Feynman graphs as implied by the perturbative nature of the calculation.

We start with the Lagrangian density of the Walecka model [1, 2]:

$$
\begin{aligned}
\mathcal{L}= & \bar{\psi}\left(i \not \partial-M+g_{s} \phi-g_{v} \gamma^{\mu} V_{\mu}\right) \psi+\frac{1}{2}\left(\partial_{\mu} \phi \partial^{\mu} \phi-m_{s}^{2} \phi^{2}\right) \\
& -\frac{1}{4} F_{\mu \nu} F^{\mu \nu}+\frac{1}{2} m_{v}^{2} V_{\mu} V^{\mu}+U(\phi, V)+\mathcal{L}_{C T}
\end{aligned}
$$

where $\psi$ represents the nucleon field operator, $\phi$ and $V_{\mu}$ are respectively the field operators of the scalar and vector meson and $F_{\mu \nu}=\partial_{\mu} V_{\nu}-\partial_{\nu} V_{\mu}$. The massive vector field is coupled to a conserved baryon current, rendering the model renormalizable in $3+1$ dimensions. In order to minimize many body effects the term $U(\phi, V)$, which describes mesonic self interactions, was set to zero in the original work of Walecka and we shall keep the same convention here. The Lagrangian density $\mathcal{L}_{C T}$ contains all the counterterms needed to render the model finite which, for the purposes of the present paper, is 
given by:

$$
\mathcal{L}_{C T}=\sum_{n=1}^{4} \frac{\beta_{n}}{n !} \phi^{n}+\zeta_{\mathrm{f}} \bar{\psi}(i \not \partial-M) \psi+M_{c} \bar{\psi} \psi
$$

We are primarily interested in studying effects of vacuum fluctuations in nuclear matter. In particular, we will consider the energy per nucleon, which is related to the energy density by:

$$
\mathcal{E}=\frac{1}{V} \int d^{3} x\left(<\Psi\left|T^{00}\right| \Psi>-<\operatorname{vac}\left|T^{00}\right| \operatorname{vac}>\right)=\mathcal{E}^{B}+\mathcal{E}^{s}+\mathcal{E}^{v},
$$

where $\mid \Psi>$ is the interacting ground-state of nuclear matter, $\mid$ vac $>$ is the vacuum state (zero density), $T^{00}$ includes the piece corresponding to $\mathcal{L}_{C T}$. The terms $\mathcal{E}^{B}, \mathcal{E}^{s}$ and $\mathcal{E}^{v}$ refer respectively to baryon, scalar-meson, and vector-meson contributions, which are given by:

$$
\begin{aligned}
\mathcal{E}^{B}= & -i \int \frac{d^{4} k}{(2 \pi)^{4}}\left\{\operatorname{Tr}\left[\gamma^{0} k^{0}-(\not k-M)\right] S(k)\right\}-\mathcal{E}_{V E V}^{B}+\mathcal{E}_{C T}^{B}, \\
\mathcal{E}^{s}= & \frac{1}{2} \frac{g_{s}^{2}}{m_{s}^{2}}\left[\int \frac{d^{4} k}{(2 \pi)^{4}} \operatorname{Tr} S(k)\right]^{2}-g_{s}^{2} \int \frac{d^{4} k}{(2 \pi)^{4}} \frac{d^{4} q}{(2 \pi)^{4}} \operatorname{Tr}[S(k+q) S(q)] \Delta_{s}\left(k^{2}\right) \\
& \times\left\{\left[\frac{1}{2}\left(k^{2}-m_{s}^{2}\right) \Delta_{s}\left(k^{2}\right)-1\right]-\left(k^{0}\right)^{2} \Delta_{s}\left(k^{2}\right)\right\}-\mathcal{E}_{V E V}^{s}+\mathcal{E}_{C T}^{s}, \\
\mathcal{E}^{v}= & -\frac{1}{2} \frac{g_{v}^{2}}{m_{v}^{2}}\left[\int \frac{d^{4} k}{(2 \pi)^{4}} \operatorname{Tr} \gamma^{0} S(k)\right]^{2}+g_{v}^{2} \int \frac{d^{4} k}{(2 \pi)^{4}} \frac{d^{4} q}{(2 \pi)^{4}} \operatorname{Tr}\left[\gamma_{\lambda} S(k+q) \gamma^{\lambda} S(q)\right] \Delta_{v}\left(k^{2}\right) \\
& \times\left\{\left[\frac{1}{2}\left(k^{2}-m_{v}^{2}\right) \Delta_{v}\left(k^{2}\right)-1\right]-\left(k^{0}\right)^{2} \Delta_{v}\left(k^{2}\right)\right\}-\mathcal{E}_{V E V}^{v}+\mathcal{E}_{C T}^{v},
\end{aligned}
$$

where $\Delta_{m}(k), m=s, v$ are the Fourier transforms of Green's functions of the KleinGordon operator. It is important to note that we are not using the nucleon equation of motion and, therefore, the above expressions differ from the usual ones [2]. The reasons for not using the nucleon equation of motion will be discussed below. The terms $\mathcal{E}_{C T}^{B, s, v}$ will be considered in renormalization of the energy density. In obtaining these expressions, we have eliminated the meson field operators $\phi$ and $V^{\mu}$ in favor of the nucleon field operators by integrating the meson Euler-Lagrange equations.

The problem of calculating the energy density consists therefore in finding the interacting nucleon propagator in medium. The first step towards the calculation of the propagator with the $\delta$ expansion is to define the interpolated Lagrangian. According to Eq. (11), one has:

$$
\mathcal{L}(\delta)=(1-\delta) \mathcal{L}_{0}+\delta \mathcal{L}=\mathcal{L}_{0}+\delta \bar{\psi}\left(g_{s} \phi-g_{v} \gamma^{\mu} V_{\mu}+\mu\right) \psi+\mathcal{L}_{C T}(\delta)
$$


where we have chosen $\mathcal{L}_{0}$ to be:

$$
\mathcal{L}_{0}=\bar{\psi}\left(i \gamma_{\mu} \partial^{\mu}-\Omega\right) \psi+\frac{1}{2}\left(\partial_{\mu} \phi \partial^{\mu} \phi-m_{\sigma}^{2} \phi^{2}\right)-\frac{1}{4} F_{\mu \nu} F^{\mu \nu}+\frac{1}{2} m_{\omega}^{2} V_{\mu} V^{\mu},
$$

where $\Omega \equiv M+\mu$. Notice that the $\delta$-expansion interpolation could also have been done in the mesonic sector. However, we have chosen to eliminate the mesonic fields by integrating their equations of motion; in this way mesonic self-energies are automatically taken into account. Of course, the integration in favor of the nucleon fields can be done only in the absence of mesonic self-interactions [Note that since we have set $U(\phi, V)=0$ the mesonic self-interactions appear in $\mathcal{L}_{C T}$ only]. This leaves us with only one unknown parameter, $\mu$, which will be fixed by the PMS condition applied to the energy density. In short, one is performing a variational calculation of the energy density with an "educated guess" for the nucleon propagator which can be improved perturbatively as higher powers of $\delta$ are considered. Since one is looking for the propagator that leaves the energy stationary, one is not allowed to substitute the nucleon equation of motion in the expression of the energy. We could also interpolate kinetic energy terms, as in Ref. [10], but we prefer to start with the simplest interpolation. Such generalizations will be examined in forthcoming publications.

The fact that the divergent part of a physical quantity $P(\mu)$ calculated with this interpolated Lagrangian will be $\delta$ and $\mu$ dependent implies that the coefficients appearing in the counterterm Lagrangian $\mathcal{L}_{C T}(\delta)$ will also depend on both parameters. The interpolated $\mathcal{L}_{C T}(\delta)$ has the same field-operator structure as the original $\mathcal{L}_{C T}$ of Eq. (3), with $\delta$ - and $\mu$-dependent coefficients. The explicit $\delta$ and $\mu$ dependence is not important since this dependence will appear automatically in the process of fixing the value of the coefficients in the renormalization process.

Next the strategy consists in calculating the interacting propagator, which is obtained by inverting Dysons's equation $S^{-1}(p)=S^{0^{-1}}(p)-\Sigma(p)$, where the self-energy $\Sigma(p)$ is calculated as a perturbation expansion in powers of $\delta$, using for the "non-interacting" propagator, $S^{0}$, the one corresponding to $\mathcal{L}_{0} . S^{0}$ can be split in the usual way as $S^{0}(k)=S_{F}^{0}(k)+S_{D}^{0}(k)$, where $S_{F}^{0}(k)$ and $S_{D}^{0}(k)$ are the Feynman and density dependent parts for quasi-particles of mass $\Omega$ and energy $E_{\Omega}=\left(\vec{k}^{2}+\Omega^{2}\right)^{1 / 2}$. Inversion of Dyson's equation is standard [2]. First, one notes that the in-medium $\Sigma(p)$ is of the general 
form [2]: $\Sigma(k)=\Sigma^{s}(k)-\gamma^{0} \Sigma^{0}(k)+\boldsymbol{\gamma} \cdot \mathbf{k} \Sigma^{v}(k)$. Then, one defines the auxiliary quantities:

$$
\begin{array}{ll}
\Omega^{*}(k)=\Omega+\Sigma^{s}(k) & \mathbf{k}^{*}=\mathbf{k}\left[1+\Sigma^{v}(k)\right], \\
E^{*}(k)=\left[\mathbf{k}^{* 2}+\Omega^{* 2}(k)\right]^{1 / 2}, & k^{* \mu}=k^{\mu}+\Sigma^{\mu}(k)=\left[k^{0}+\Sigma^{0}(k), \mathbf{k}^{*}\right] .
\end{array}
$$

The propagator can be written as $S(k)=S_{F}(k)+S_{D}(k)$, with:

$$
S_{F}(k)=\frac{1}{\not k^{*}-\Omega^{*}(k)+i \epsilon}, \quad S_{D}(k)=i \pi \frac{\not k^{*}+\Omega^{*}(k)}{E^{*}(k)} \delta\left(k^{0}-E(k)\right) \theta\left(k_{F}-|\mathbf{k}|\right),
$$

where $E(k)$ is the single-particle energy, which satisfies $E(k)=\left[E^{*}(k)-\Sigma^{0}(k)\right]_{k^{0}=E(k)}$. Note that we have assumed that the nucleon propagator has simple poles with unit residue. Within the approximation scheme we will work in this paper, this assumption is satisfied, as can be seen below.

The renormalization procedure in the $\delta$ expansion follows closely the usual perturbative renormalization program, with small, however important, differences. Hence, in the following we shall repeat some standard textbook material which we feel essential for the appreciation of the differences. We start with the problem in vacuum $\left(k_{F}=0\right)$, in which case $S^{0}(k)=S_{F}^{0}(k)$. We perform our calculations in $2 \omega=4-2 \epsilon$ dimensions using dimensional regularization techniques [1]. To $\mathcal{O}(\delta)$, the self-energy is simply $\Sigma_{F}^{(1)}(k)=-\delta \mu$, which arises from the bilinear term $\delta \mu \bar{\psi} \psi$. To $\mathrm{O}\left(\delta^{2}\right)$, one has in principle tadpole and exchange contributions:

$$
\Sigma_{F}^{(2) \operatorname{tad}}(k)=i \delta^{2}\left(\frac{g_{s}}{m_{s}}\right)^{2} \int \frac{d^{4} q}{(2 \pi)^{4}} \operatorname{Tr}\left[S_{F}^{0}(q)\right]-i \delta^{2}\left(\frac{g_{v}}{m_{v}}\right)^{2} \int \frac{d^{4} q}{(2 \pi)^{4}} \gamma_{\mu} \operatorname{Tr}\left[\gamma^{\mu} S_{F}^{0}(q)\right],
$$

and

$$
\Sigma_{F}^{(2) \operatorname{exch}}(k)=i \delta^{2} g_{s}^{2} \int \frac{d^{4} q}{(2 \pi)^{4}} S_{F}^{(0)}(q) \Delta_{s}(k-q)-i \delta^{2} g_{v}^{2} \int \frac{d^{4} q}{(2 \pi)^{4}} \gamma_{\mu} S_{F}^{(0)}(q) \Delta_{v}(k-q) \gamma^{\mu} .
$$

The vector meson tadpole contribution vanishes after taking the trace and performing the integral, whereas scalar-meson tadpole contains a finite and a divergent part. The renormalization of this is performed using the term $\beta_{1}(\delta) \phi$ from $\mathcal{L}_{C T}(\delta)$ in Eq. (3). Its contribution to the self-energy is $\Sigma_{\beta_{1}}=\beta_{1}(\delta) g_{s} \Delta_{s}(0)$. In order to produce a stable vacuum, $\beta_{1}(\delta)$ is chosen so as to eliminate the divergent and finite parts. Any finite piece must be canceled, since such a term would contribute to the energy of the vacuum, which in turn could be lowered without bound. 
The exchange parts of the self-energy are given by:

$$
\Sigma_{F}^{(2) \operatorname{exch}}(k)=-\delta^{2}\left[\not k a\left(k^{2}\right)-b\left(k^{2}\right)\right]
$$

where

$$
\begin{aligned}
& a\left(k^{2}\right)=\frac{g_{s}^{2}}{(4 \pi)^{2}}\left[\frac{1}{\epsilon}-I_{0}^{s}\left(k^{2}\right)\right]+\frac{2 g_{v}^{2}}{(4 \pi)^{2}}\left[\frac{1}{\epsilon}-I_{0}^{v}\left(k^{2}\right)\right]+C_{a}, \\
& b\left(k^{2}\right)=-\frac{g_{s}^{2} \Omega}{(4 \pi)^{2}}\left[\frac{1}{\epsilon}-I_{1}^{s}\left(k^{2}\right)\right]+\frac{4 g_{v}^{2} \Omega}{(4 \pi)^{2}}\left[\frac{1}{\epsilon}-I_{1}^{v}\left(k^{2}\right)\right]+C_{b},
\end{aligned}
$$

where $C_{a}$ and $C_{b}$ are irrelevant constants and $I_{i}^{m}\left(k^{2}\right), \quad i=1,2$ are the integrals:

$$
I_{i}^{m}\left(k^{2}\right)=\int_{0}^{1}(d \alpha)_{i} \ln \left[\frac{\left.k^{2}(\alpha-1) \alpha+(1-\alpha) \Omega^{2}+\alpha m_{m}^{2}\right)}{m_{m}^{2}}\right],
$$

where $(d \alpha)_{0}=\alpha d \alpha$ and $(d \alpha)_{1}=d \alpha$, with $\alpha$ being the Feynman parameter.

The renormalization of the exchange self-energy is carried out with the last two terms of Eq, (3), which contribute to the self-energy as $\Sigma_{F}^{\mathrm{CT}}(k)=-M_{c}(\delta)+\zeta_{\mathrm{f}}(\delta)(\not k-M)$, and the renormalized vacuum contribution to the self-energy, $\Sigma_{F}^{R}(k)$, up to $\mathcal{O}\left(\delta^{2}\right)$ is given by $\Sigma_{F}^{R}(k)=-\delta \mu+\Sigma_{F}^{(2) \operatorname{exch}}(k)+\Sigma_{F}^{\mathrm{CT}}(k)$.

Generally, counterterms are composed of a divergent part which completely eliminates the poles and of an arbitrary finite part which is fixed according to a chosen renormalization scheme [11. Here we shall consider the parameters $g_{m}, m_{m}$ and $M$ to be "the" renormalized physical couplings and masses. This choice amounts to the on-mass shell renormalization scheme in which the counterterms remove both divergent and finite contributions from loop corrections to measurable amplitudes. Within this renormalization scheme the finite parts of both counterterms are fixed by the renormalization conditions:

$$
\left.S_{F}^{-1}(k)\right|_{k=M}=\left.\left[S_{F}^{0^{-1}}(k)-\Sigma_{F}^{R}(k)\right]\right|_{k=M}=0 \quad \text { and }\left.\quad \frac{\partial S_{F}^{-1}(k)}{\partial \not k}\right|_{k=M}=1 .
$$

Application of these conditions leads to the following expression for the renormalized self-energy in vacuum:

$$
\Sigma_{F}^{R}(k)=A\left(k^{2}\right) \not k-B\left(k^{2}\right),
$$

where the functions $A\left(k^{2}\right)$ and $B\left(k^{2}\right)$ are free of divergencies and given by:

$$
A\left(k^{2}\right)=-\delta^{2}\left[\bar{a}\left(k^{2}\right)-2 M \bar{c}\left(M^{2}\right)\right], \quad B\left(k^{2}\right)=\mu-\delta^{2}\left[\bar{b}\left(k^{2}\right)-2 M^{2} \bar{c}\left(M^{2}\right)\right],
$$


with $\bar{a}\left(k^{2}\right)=a\left(k^{2}\right)-a\left(M^{2}\right), \bar{b}\left(k^{2}\right)=b\left(k^{2}\right)-b\left(M^{2}\right)$, and $\bar{c}\left(M^{2}\right)=M \bar{a}^{\prime}\left(M^{2}\right)-\bar{b}^{\prime}\left(M^{2}\right)$, where the prime denotes a derivative with respect to the argument. It is easy to check that Eqs. (15) are indeed satisfied by Eqs. (16-17).

In principle one can proceed to higher orders in $\delta$ and include, for example, the important vertex corrections. The renormalization at higher orders introduces no extra complications as compared to the usual perturbative renormalization. Instead of going to higher orders, we consider next the problem of the energy density, where new divergencies arise and extra renormalization is required. In order to simplify the discussion, we shall consider here only direct terms and then compare the results with the ones obtained within the RHA.

When considering direct terms only, up to $\mathcal{O}\left(\delta^{2}\right)$ one obtains the following relations for the auxiliary quantities defined in Eq. (8):

$$
\begin{aligned}
& \Omega^{*}(k)=\Omega-\delta \mu-\delta^{2} 4 \frac{g_{\sigma}^{2}}{m_{\sigma}^{2}} \int_{0}^{k_{F}} \frac{d^{3} q}{(2 \pi)^{3}} \frac{\Omega}{E_{\Omega}(q)}, \\
& k^{0 *}=k^{0}-\delta^{2} 4 \frac{g_{\omega}^{2}}{m_{\omega}^{2}} \int_{0}^{k_{F}} \frac{d^{3} q}{(2 \pi)^{3}}, \quad \mathbf{k}^{*}=\mathbf{k}
\end{aligned}
$$

From these, one constructs the nucleon propagator which is then used in the expression for the energy density. We start with the baryon contribution to the energy density:

$$
\begin{aligned}
\mathcal{E}^{B} & =-\frac{3 \Omega^{* 4}}{8 \pi^{2}}\left[\frac{1}{\epsilon}+\psi(3)+\ln \left(\frac{4 \pi \eta^{2}}{\Omega^{* 2}}\right)\right]+\frac{M \Omega^{* 3}}{2 \pi^{2}}\left[\frac{1}{\epsilon}+\psi(2)+\ln \left(\frac{4 \pi \eta^{2}}{\Omega^{* 2}}\right)\right] \\
& +4 \int_{0}^{k_{F}} \frac{d^{3} \vec{k}}{(2 \pi)^{3}} \frac{\vec{k}^{2}+M \Omega^{*}}{E^{*}(k)}-\mathcal{E}_{V E V}^{B}+\mathcal{E}_{C T}^{B} .
\end{aligned}
$$

Note that the divergent and finite parts are different from the usual ones [2] because we have not used the nucleon field equation of motion. Carrying out the VEV subtraction, the remaining divergencies are eliminated by the counterterm $\sum_{n=2}^{4}\left(\beta_{n} / n !\right) \phi^{n}$ in Eq. (3). Thus, the finite contribution to the baryonic part of the energy density is given by (after taking $\delta=1)$ :

$$
\mathcal{E}^{B}=\int_{0}^{k_{F}} \frac{d^{3} \vec{k}}{(2 \pi)^{3}} \frac{\mathbf{k}^{2}+M \Omega^{*}}{E^{*}(k)}+\Delta_{B},
$$

where

$$
\begin{aligned}
\Delta_{B} & =\frac{1}{4 \pi^{2}}\left\{\ln \left(\frac{\Omega^{*}}{M}\right)\left[3\left(\Omega^{*}\right)^{4}-4 M\left(\Omega^{*}\right)^{3}\right]\right. \\
& \left.+M^{3}\left(\Omega^{*}-M\right)-\frac{1}{2} M^{2}\left(\Omega^{*}-M\right)^{2}-\frac{17}{3} M\left(\Omega^{*}-M\right)^{3}-\frac{21}{4}\left(\Omega^{*}-M\right)^{4}\right\} .
\end{aligned}
$$


Proceeding in the same way for the scalar meson contribution, we obtain:

$$
\mathcal{E}^{s}=-\frac{m_{s}^{2}}{2 g_{s}^{2}}\left(4 \frac{g_{s}^{2}}{m_{s}^{2}} \int \frac{d^{3} k}{(2 \pi)^{3}} \frac{\Omega^{*}}{E^{*}(k)}-\Delta_{S}\right)^{2}
$$

where

$$
\Delta_{S}=\frac{g_{s}^{2}}{m_{s}^{2}} \frac{1}{\pi^{2}}\left[\Omega^{* 3} \ln \left(\frac{\Omega^{*}}{M}\right)-M^{2}\left(\Omega^{*}-M\right)-\frac{5}{2} M\left(\Omega^{*}-M\right)^{2}-\frac{11}{6}\left(\Omega^{*}-M\right)^{3}\right] .
$$

Finally, there are no divergencies with the vector meson contribution, it is given by:

$$
\mathcal{E}^{v}=\frac{1}{2} \frac{g_{v}^{2}}{m_{v}^{2}}\left[\frac{2}{3 \pi^{2}} k_{F}^{3}\right]^{2} .
$$

The determination of the unknown parameter $\mu$ follows from the PMS applied to the energy density:

$$
\frac{d \mathcal{E}}{d \mu}=\frac{d \mathcal{E}}{d \Omega^{*}} \frac{d \Omega^{*}}{d \mu}=0 \Rightarrow \frac{d \mathcal{E}}{d \Omega^{*}}=0,
$$

where we used the fact that $d \Omega^{*} / d \mu \neq 0$. Differentiation of $\mathcal{E}$ with respect to $\Omega^{*}$ leads to the following self-consistent equation for the effective nucleon mass $\Omega^{*}$ :

$$
\left\{\left(M-\Omega^{*}\right)-\left[4 \frac{g_{s}^{2}}{m_{s}^{2}} \int \frac{d^{3} k}{(2 \pi)^{3}} \frac{\Omega^{*}}{E^{*}(k)}-\Delta_{S}\right]\right\}\left[4 \frac{g_{s}^{2}}{m_{s}^{2}} \int \frac{d^{3} k}{(2 \pi)^{3}} \frac{\mathbf{k}^{2}}{E^{* 3}(k)}-\frac{d \Delta_{S}}{d \Omega^{*}}\right]=0 .
$$

To arrive at this result we made use of the following identity:

$$
\frac{d \Delta_{B}}{d \Omega^{*}}=\left(\Omega^{*}-M\right) \frac{m_{s}^{2}}{g_{s}^{2}} \frac{d \Delta_{S}}{d \Omega^{*}}
$$

Clearly, Eq. (27) admits two solutions. The one that follows from the vanishing term in curly braces corresponds to the usual RHA self-consistent solution. It is not difficult to show that when this is substituted into the equation for the energy density, one obtains the RHA energy density:

$$
\mathcal{E}=\frac{g_{v}^{2}}{2 m_{v}^{2}}\left(\frac{2}{3 \pi^{2}} k_{F}^{3}\right)^{2}+\frac{m_{s}^{2}}{2 g_{s}^{2}}\left(\Omega^{*}-M\right)^{2}+4 \frac{g_{s}^{2}}{m_{s}^{2}} \int \frac{d^{3} k}{(2 \pi)^{3}} E^{*}(k)+\Delta \mathcal{E}_{V F}
$$

where $\Delta \mathcal{E}_{V F}$ is the energy density corresponding to vacuum fluctuations, given by:

$$
\begin{aligned}
\Delta \mathcal{E}_{V F} & =\frac{1}{4 \pi^{2}}\left[-\Omega^{* 4} \ln \left(\frac{\Omega^{*}}{M}\right)+M^{3}\left(\Omega^{*}-M\right)\right. \\
& \left.+\frac{7}{2} M^{2}\left(\Omega^{*}-M\right)^{2}+\frac{13}{3}\left(\Omega^{*}-M\right)^{3}+\frac{25}{12}\left(\Omega^{*}-M\right)^{4}\right] .
\end{aligned}
$$


With the parameter set [Q], $g_{s}^{2}=62.89, g_{v}^{2}=79.78, m_{s}=550 \mathrm{MeV}, m_{v}=783 \mathrm{MeV}$ and $M=939 \mathrm{MeV}$, nuclear matter is saturated at $k_{F}=1.42 \mathrm{fm}^{-1}$, with a binding energy of $16 \mathrm{MeV}$ per nucleon.

The other solution to Eq. (27) is:

$$
\frac{d \Delta_{S}}{d \Omega^{*}}=4 \frac{g_{s}^{2}}{m_{s}^{2}} \int \frac{d^{3} k}{(2 \pi)^{3}} \frac{\mathbf{k}^{2}}{E^{* 3}(k)} .
$$

Note that the solutions of this equation are independent of the parameters of the model; the effective nucleon mass $\Omega^{*}$ depends only on $M$ and $k_{F}$. The numerical solution of this equation gives an effective nucleon mass that is an increasing function of the nuclear density. Moreover, when this solution is substituted into the original expression for the energy density, and using the same parameters as the ones of the RHA, we find a new saturation point for nuclear matter at $k_{F} \sim 5.3 \mathrm{fm}^{-1}$, which corresponds to a density of approximately 50 times the one of normal nuclear matter, with a binding energy of the order of $570 \mathrm{MeV}$ per nucleon. Of course, if the parameters are changed, these numbers will change. However, we do not pursue the discussion on this further because exchange terms should be included for consistency (they are of the same order in $\delta$ as the direct ones), although one should notice that in all known cases where exchange graphs are included their effect can be absorbed by a readjustment of parameters [2].

When one considers the exchange diagrams in the energy density, the renormalization becomes more difficult, but not more complicated than the usual renormalization in the loop expansion [12]. In particular, if instead of inverting Dyson's equation for obtaining the propagator from the self-energy one uses the same approach as Chin [13] for treating the exchange diagrams, the renormalization is indeed simplified.

We have given the first step towards the implementation of the $\delta$-expansion for studying vacuum effects in effective hadron field theories. We demonstrated how the renormalization program can be implemented within the $\delta$ expansion. We also calculated the renormalized energy density of nuclear matter at $\mathcal{O}\left(\delta^{2}\right)$ by neglecting exchange diagrams, and found that the usual RHA approximation is readily reproduced. In addition, a new state of nuclear matter is found from the PMS applied to the renormalized energy density. Concluding, we believe that the results are very encouraging for future investigations concerning the vacuum of hadronic models. 
This work was partially supported by CNPq and FAPESP (contract \# 93/2463-2). The authors would like to thank Marina Nielsen and MBP would like to thank P. Grangé and G. Mennessier for helpful discussions.

\section{References}

[1] J.D. Walecka, Ann. Phys. (N.Y.) 83 (1974) 491.

[2] B.D. Serot and J.D. Walecka, Adv. Nucl. Phys. 16, 1 (1986).

[3] A. Bielajew and B.D. Serot, Ann. Phys. (N.Y.) 156 (1984) 215.

[4] C.M. Bender, K.A. Milton, M. Moshe, S.S. Pinsky and L.M. Simmons Jr., Phys., Rev. Lett. 58 (1987) 2615 .

[5] A. Okopińska, Phys. Rev. D 35 (1987) 1835.

[6] M.B. Pinto, Phys. Rev. D 50 (1994) 7673.

[7] G. Krein, D.P. Menezes, M. Nielsen and M.B. Pinto, submitted to publication; electronic archive hep-ph/9505216.

[8] P.M. Stevenson, Phys. Rev. D 23 (1981) 2916.

[9] For a list of references see Ref. [6], [10].

[10] D. Gromes, preprint HD-THEP-95-38; electronic archive hep-ph/9509224

[11] J.C. Collins, Renormalization (Cambridge University Press, New York, 1984).

[12] R.J. Furnstahl, R.J. Perry and B.D. Serot, Phys. Rev. C 40 (1989) 321.

[13] S.A. Chin, Ann. Phys. (NY) 108 (1977) 301. 